\title{
Is the intercellular adhesion molecule-1/leukocyte function associated antigen 1 pathway of leukocyte adhesion involved in the tissue damage of alcoholic hepatitis?
}

\author{
P Burra, S G Hubscher, J Shaw, E Elias, D H Adams
}

\begin{abstract}
Alcoholic hepatitis is characterised histologically by an intense inflammatory cell infiltrate made up predominantly of neutrophils but including other cell types, particulary lymphocytes. Leukocyte cytotoxicity requires cell adhesion, which is mediated via receptors on the leukocyte surface including leukocyte function associated antigen-1 (LFA-1) which binds to the ligand intercellular adhesion molecule-1 (ICAM-1) on the target cell. The distribution of ICAM-1 and LFA-1 expression in liver biopsy specimens from patients with alcoholic liver disease was examined to ascertain whether this pathway of leukocyte adhesion is involved in the tissue damage of alcoholic hepatitis. Specimens were stained for ICAM-1 and LFA-1 by a three step immunoalkalinephosphatase method using monoclonal antibodies against ICAM-1 and LFA-1. LFA-1 staining on portal tract inflammatory cells and parenchymal inflammatory cells and ICAM-1 staining on liver components were examined. ICAM-1 expression on hepatocytes was significantly greater in alcoholic hepatitis compared with fatty liver $(p<0.001)$ and normal controls $(\mathbf{p}<0.01)$. ICAM-1 expression correlated with the histological degree of hepatocellular damage (tau $=0.79 ; p=0.0005)$ and parenchymal inflammation (tau $=0.65 ; p<0.001$, and with LFA-1 expression on parenchymal leukocytes $(\operatorname{tau}=0.63 ; p=0.01)$. The ICAM-1/LFA-1 pathway may therefore be involved in leukocyte mediated tissue damage during alcoholic hepatitis.
\end{abstract}

The pathogenesis of liver damage in alcoholic hepatitis is not well understood, although there is evidence implicating immune mechanisms. Liver biopsy tissue shows inflammation, which may be focal or diffuse in distribution and is associated with hepatocellular necrosis, and ballooning of hepatocytes suggesting that there is leukocyte mediated tissue damage. The cellular infiltrate consists predominantly of neutrophils,' although other cell types such as lymphocytes are also seen. ${ }^{2}$

The induction and maintenance of leukocyte cytotoxicity requires cell adhesion, both to target cells and to other immune cells. Adhesion is mediated via a number of receptors on the leukocyte surface including leukocyte function associated antigen-1 (LFA-1), a member of the CD18 family of adhesion molecules, which binds to intercellular adhesion molecule-1 (ICAM-1) on target cells. ${ }^{3}$ ICAM-1 is a cell surface glycoprotein which is expressed on endothelial cells, epithelial cells, fibroblasts, and haematopoetic cells. Expression can be increased in vitro by proinflammatory cytokines such as interferon $\gamma$ ( $\gamma$ IFN), interleukin 1 (IL-1), and tumour necrosis factor (TNF) ${ }^{47}$

This study examines the distribution of ICAM-1/LFA-1 expression in liver biopsy specimens from patients with alcoholic liver disease in order to investigate whether cell adhesion molecules play a role in the pathogenesis of alcoholic hepatitis.

\section{Methods}

Twenty five liver biopsy specimens were collected from patients with alcoholic liver disease and 13 control samples were taken from donor livers at the time of liver transplantation. Biopsy samples were grouped according to the morphological criteria of alcoholic liver disease as described by the International Liver Study Group. ${ }^{8}$ Eight patients were thereby classified as fatty liver (FC), 10 had alcoholic hepatitis $(\mathrm{AH})$, and seven cirrhosis (AC). All of the alcoholic patients were drinking up to the time of admission to hospital.

\section{BIOPSY SPECIMENS}

Biopsy specimens were snap frozen in liquid nitrogen and stored at $-70^{\circ} \mathrm{C}$. After thawing, sections were fixed in acetone and stained for ICAM-1 and LFA-1 by means of a three step immunoalkaline-phosphatase method as previously described ${ }^{9}$ using mouse monoclonal antibodies recognising ICAM-1 and LFA-1 (gift from $\operatorname{Dr} \mathbf{R}$ Rothlein, Boehringer Ingelheim, Ridgefield, Connecticut, USA) diluted 1/5000 in TRIS buffered saline.

Sections were examined by two observers (DHA, SGH) who were unaware of the patients' clinical condition or histological status. Both the 
TABLE I Histological scores for portal and parenchymal inflammation and for hepatocellular damage for the three groups of patients (results mean (SEM)).

\begin{tabular}{llll}
\hline & Fatty liver & $\begin{array}{l}\text { Alcoholic } \\
\text { hepatitis }\end{array}$ & Cirrhosis \\
\hline Inflammation: & & & \\
$\quad$ Portal & $0.81(0.23)$ & $1.25(0.20)$ & $1.42(0.29)$ \\
$\quad$ Parenchymal & $0 \cdot 75(0 \cdot 16)$ & $1.50(0.30)$ & $0.85(0 \cdot 14)$ \\
Hepatocellular damage & $2.31(0.38)$ & $6.65(0.87)$ & $4 \cdot 14(0.85)$ \\
\hline
\end{tabular}

TABLE II Median (range) staining score for leukocyte function associated antigen-1 $(L F A-1)$ expression on portal tract and parenchymal inflammatory infiltrate and intercellular adhesion molecule-1 (ICAM-1) expression on hepatocyte membranes in patients with fatty liver, alcoholic hepatitis, and alcoholic cirrhosis and in control subjects

\begin{tabular}{|c|c|c|c|}
\hline & \multicolumn{2}{|c|}{ Inflammatory infiltration } & \multirow[b]{2}{*}{$\begin{array}{l}\text { Hepatocytes } \\
\text { ICAM-1 }\end{array}$} \\
\hline & $\begin{array}{l}\text { Portal tract } \\
\text { LFA-I }\end{array}$ & $\begin{array}{l}\text { Parenchymal } \\
\text { LFA-I }\end{array}$ & \\
\hline $\begin{array}{l}\text { Fatty liver } \\
\qquad(\mathrm{n}=8)\end{array}$ & $1 \cdot 75(0-3 \cdot 0)$ & $1 \cdot 0(1 \cdot 0-3 \cdot 0)$ & $0 \cdot 75(0 \cdot 5-2 \cdot 0)$ \\
\hline $\begin{array}{l}\text { Alcoholic hepatitis } \\
(\mathrm{n}=10)\end{array}$ & $2 \cdot 0(1 \cdot 5-2 \cdot 5)$ & $2.0(1.0-3 \cdot 0) \dagger$ & $3.0(0.5-3.0) \ddagger$ \\
\hline $\begin{array}{l}\text { Alcoholic cirrhosis } \\
(\mathrm{n}=7)\end{array}$ & $2 \cdot 5(2 \cdot 5-3 \cdot 0)^{\star}$ & $1 \cdot 0(0 \cdot 5-2 \cdot 5)$ & $2 \cdot 0(0 \cdot 5-3 \cdot 0)$ \\
\hline $\begin{array}{l}\text { Control subjects } \\
\quad(n=13)\end{array}$ & & & $1 \cdot 0(0-2 \cdot 5)$ \\
\hline
\end{tabular}

$\star \mathrm{p}=0.008 v$ fatty liver; $\mathrm{p}=0.001 v$ alcoholic hepatitis. $\mathrm{p}=0.008 v$ fatty liver; $\mathrm{p}=0.001$
$\mathrm{p}=0.045 v$ alcoholic cirrhosis.

$\dagger \mathrm{p}=0.045 v$ alcoholic cirrhosis.
$\ddagger \mathrm{p}=0.004 v$ fatty liver; $\mathrm{p}=0.01 v$ control.

portal tract and the parenchymal inflammatory infiltrate were examined for LFA-1 staining; hepatocytes, bile ducts, Kupffer cells, and inflammatory cells were examined for ICAM-1 staining. Staining on each structure was scored on a semiquantitative scale of $0-3$ according to its extent and intensity.

The following histological features were assessed independently on conventionally stained tissue sections by two observers (PB, SGH): hepatocyte ballooning, intracytoplasmic Mallory bodies, fatty change, necrosis, cholestasis, fibrosis and inflammation in the parenchyma, and inflammation and fibrosis in the portal tracts. Each of these features was also scored on a semiquantitative scale of $0-3$. A 'hepatocellular damage score' was obtained by

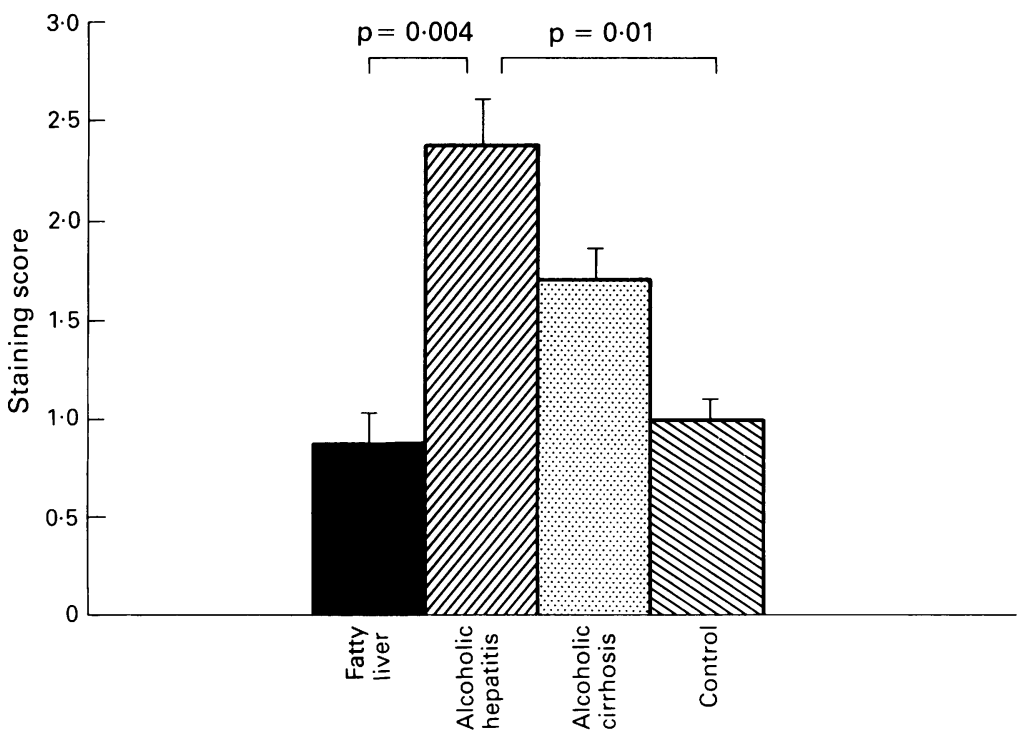

Figure 1: Intercellular adhesion molecule-1 expression on hepatocyte membranes is significantly greater in alcoholic hepatitis than in fatty liver and normal control subjects. The staining score, on a scale 0-3, is expressed as mean (SEM).

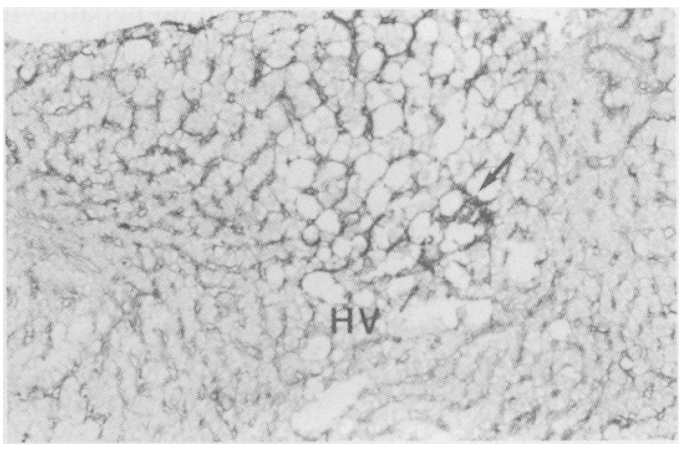

Figure 2: Strong membranous staining for intercellular adhesion molecule-1 (ICAM-1) is seen in ballooned perivenular hepatocytes. Elsewhere, hepatocyte staining is weak or absent. A small collection of inflammatory cells (arrowed) also stains positively for ICAM-1. There is diffuse staining of sinusoidal lining cells (Kupffer cells). ( $H V=$ hepatic venule) (Immunoalkaline phosphatase, original magnification, x130.)

calculating the mean scores of five of the parenchymal features listed above: ballooning, Mallory bodies, fatty change, necrosis, and cholestasis. An 'inflammation score' was obtained by calculating the mean of the inflammatory cell scores in parenchyma and portal tracts (Table I).

Results were compared by the Mann-Whitney $\mathrm{U}$ test for non-parametric data taking a p value of $<0.01$ as significant. Correlations were made by Kendall tau correlation analysis.

\section{Results}

\section{HISTOLOGICAL FEATURES}

The hepatocellular damage scores and the inflammation scores for the different groups are shown in table $\mathbf{I}$.

\section{ICAM-1 EXPRESSION ON HEPATOCYTE} MEMBRANES

Increased ICAM-1 expression was detected on hepatocyte membranes in patients with alcoholic hepatitis compared with normal controls $(p=0.01)$ and those with fatty liver $(p=0.004)$. The staining score for specimens from patients with alcoholic hepatitis was higher than that for patients with cirrhosis but the difference did not reach statistical significance (Fig 1). The distribution of staining was mainly perivenular and

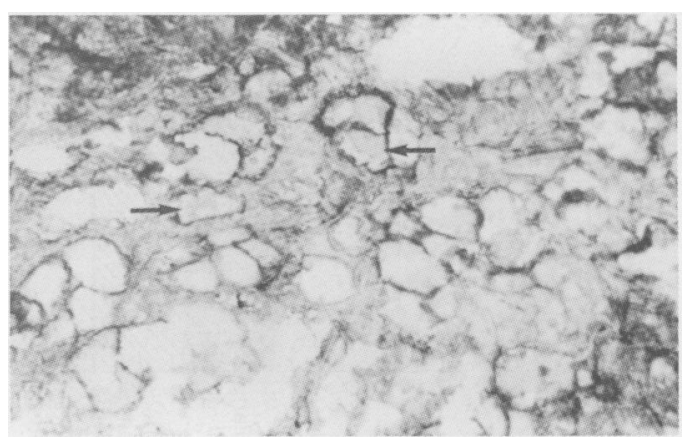

Figure 3: Higher power showing strong membranous staining of ballooned hepatocytes in an area of perivenular fibrosis. Many of the ballooned hepatocvtes contain deposits of

Mallory's hyalin (arrowed). (Immunoalkaline phosphatase, original magnification, $\times 330$.) 
was seen on hepatocytes showing morphological damage (Figs 2 and 3). Infiltrating leukocytes staining for ICAM-1 were also seen in these areas (Fig 2). In patients with alcoholic cirrhosis, the

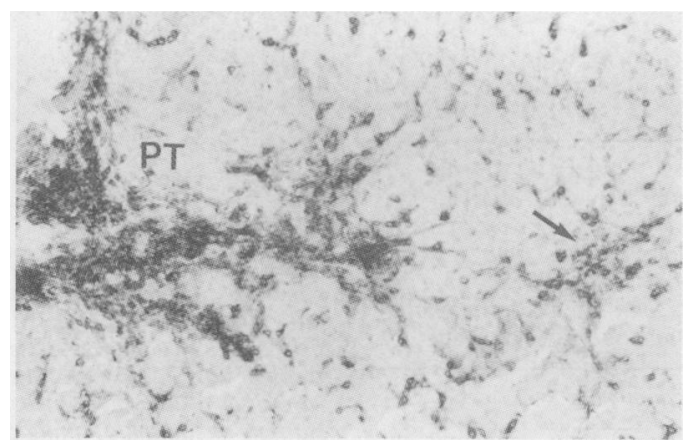

Figure 4: Leukocyte function associated antigen-1 staining in portal and parenchymal inflammatory cells. Parenchymal staining is present in a diffuse sinusoidal distribution with formation of a small inflammatory aggregate (arrowed) in a perivenular region. ( $P T=$ portal tract $)$ (Immunoalkaline phosphatase, original magnification, x130.)

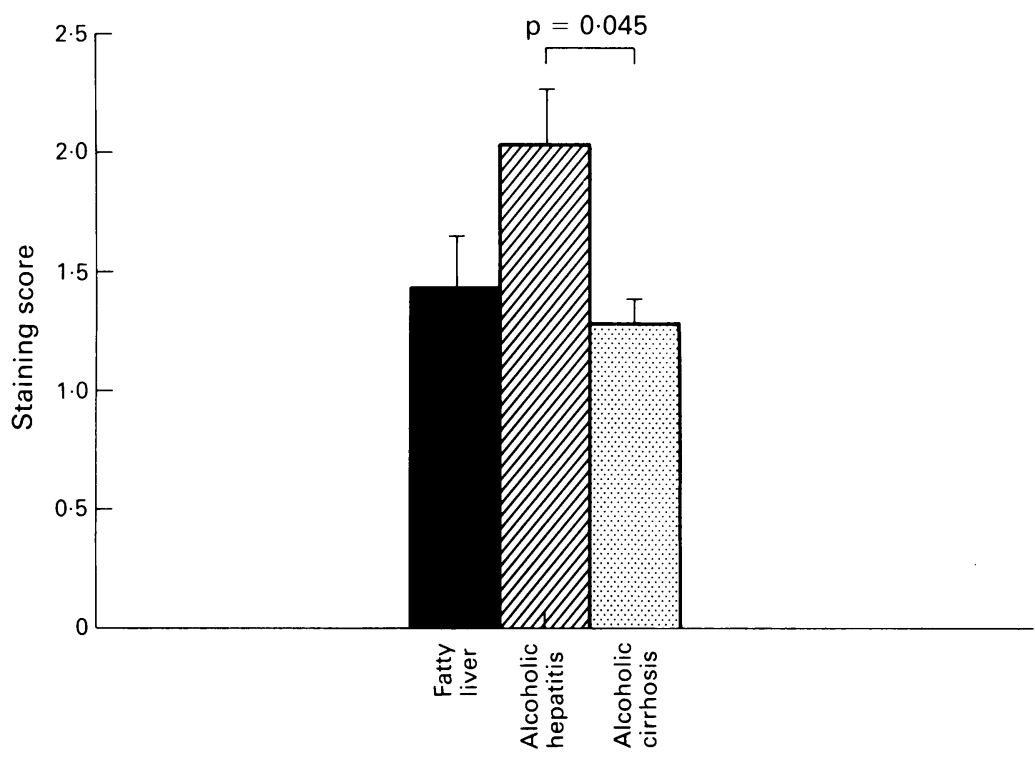

Figure 5: Leukocyte function associated antigen-1 expression on parenchymal inflammatory infiltrate for the three patient groups. The staining score is on a scale $0-3$, results are given as mean (SEM).

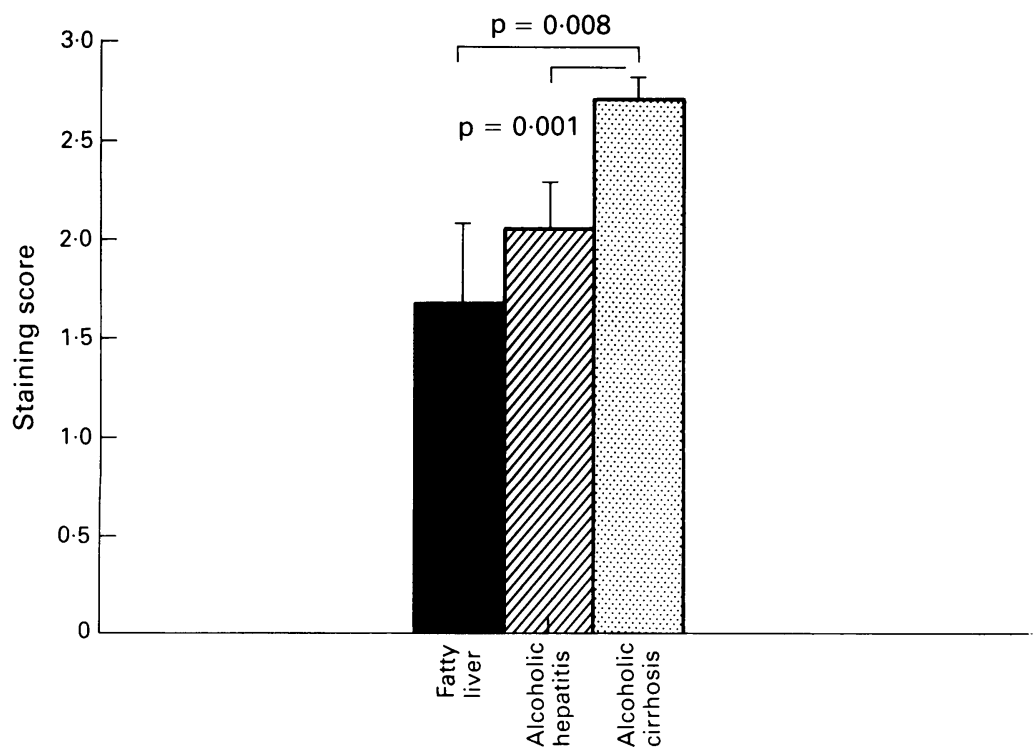

Figure 6: Leukocyte function associated antigen-1 expression on portal tract inflammatory. infiltrate is significantly greater in alcoholic cirrhosis than in fatty lizer. The staining score is on a scale 0-3 and results are given as mean (SEM). staining pattern was different, being mostly periseptal, a pattern of staining previously associated with cirrhosis from other causes."

ICAM-1 expression on hepatocytes in alcoholic hepatitis correlated with parenchymal inflammation ( $\operatorname{tau}=0.65 ; \mathrm{p}=0.0008), \mathrm{LFA}-1$ expression on parenchymal leukocytes $(\operatorname{tau}=0.63 ; \mathrm{p}=0.01)$, and histological features of hepatocellular damage $(\operatorname{tau}=0 \cdot 79 ; \mathrm{p}=0.0005)$. The hepatocyte expression in alcholic cirrhosis did not correlate with inflammation, hepatocyte damage, or LFA-1 expression.

Bile duct staining for ICAM-1 was generally weak or absent and no significant differences were seen between the groups, Kupffer cells showed strongly positive staining for ICAM-1 (Fig 2) in all biopsy specimens. There were no significant differences between the groups.

\section{LFA-1 EXPRESSION ON INFILTRATING \\ LEUKOCYTES}

Many inflammatory cells in portal tracts and liver parenchyma showed positive staining for LFA-1 (Fig 4). In alcoholic hepatitis most of the cells that stained for LFA-1 were neutrophils and were found predominantly in the parenchyma. In alcoholic hepatitis the median staining score for LFA-1 on parenchymal leukocytes was greater than that for alcoholic cirrhosis, although the difference was only of borderline statistical significance $(p=0.045)$ (Fig 5). In alcoholic cirrhosis, LFA-1 expression on portal tract leukocytes was significantly greater than in fatty liver $(\mathrm{p}=0.008)$ (Fig 6).

No correlation was found between the histological severity of the portal tract infiltrate and LFA-1 expression on portal tract leukocytes. A significant correlation was found, however, between the histological severity of the parenchymal infiltrate and LFA-1 expression on parenchymal leukocytes in alcoholic hepatitis $(\mathrm{tau}=0.58 ; \mathrm{p}=0.0096)$.

\section{Discussion}

In the normal liver, ICAM-1 expression is largely confined to sinusoidal lining cells with only faint staining on hepatocyte membranes. In contrast, the present study shows that in alcoholic hepatitis ICAM-1 is strongly expressed on hepatocytes, particularly in areas of inflammatory damage. These findings support the hypothesis that immunological mechanisms have a role in the hepatocellular damage of alcoholic hepatitis. Furthermore, the finding that the infiltrating leukocytes express LFA-1 (the receptor for ICAM-1) indicates that they would be capable of adhering to and damaging hepatocytes in alcoholic hepatitis. In contrast, patients with alcoholic cirrhosis showed a different pattern of hepatocyte staining. This was largely confined to the periseptal areas and was not associated with inflammation or hepatocyte damage. This pattern of hepatocyte staining has been described previously in patients with cirrhosis due to other causes and may be related to the process of fibrogenesis." "11

ICAM-1 expression has been shown on target structures in a number of inflammatory liver 
conditions $\mathrm{s}^{910}$ but the factors leading to increased ICAM-1 expression on hepatocytes are unknown. Since proinflammatory cytokines are capable of enhancing ICAM-1 expression on cell lines in vitro, ${ }^{4}$ locally released cytokines may play a part. The signal for cytokine release in alcoholic hepatitis are unclear but could be a factor derived from alcohol related liver damage which stimulates immune activation and cytokine release. This antigen may be alcoholic hyalin, which can cause lymphocyte activation ${ }^{12-14}$ and can also act as a chemotactic factor and stimulant for polymorphonuclear leukocytes. Furthermore, both tumour necrosis factor (TNF) production by monocytes $^{15}$ and interleukin-1 release ${ }^{16}$ are increased in alcoholic hepatitis and both these cytokines are potent inducers of ICAM-1 expression in vitro. Patients with alcoholic liver disease frequently have endotoxinaemia, a major stimulus for TNF production. ${ }^{17} 18$ The release of TNF and other proinflammatory cytokines in response to either endotoxinaemia or alcoholic hyalin, or both, could, therefore lead to the increased expression of adhesion molecules in alcoholic hepatitis. Furthermore, proinflammatory cytokines can both activate neutrophils and recruit them to areas of inflammation. Such activation results in an increased expression of LFA-1 on the neutrophil membrane which would facilitate adhesion to hepatocytes expressing ICAM-1. CD8 T cells, which have been implicated in the hepatocyte damage of alcoholic hepatitis, ${ }^{19}$ also adhere via the ICAM-1/LFA-1 pathway and could therefore also interact with ICAM-1 on hepatocytes.

The results of the present study have potentially important therapeutic implications. Corticosteroids have been shown to diminish ICAM1 expression during rejection of liver allografts ${ }^{9}$ and it is therefore possible that a similar mechanism might explain the improved survival of patients with severe alcoholic hepatitis when treated with corticosteroids. ${ }^{20}$ Furthermore, therapeutic antibodies to ICAM-1, which are currently being developed ( $\mathrm{R}$ Rothlein personal communication), may have a role in the treatment of severe alcoholic hepatitis, a condition which at present has a high mortality. ${ }^{1}$
We thank Dr Robert Rothlein, Boehringer Ingelheim Pharmaceuticals Inc, Ridgefield, Connecticut, USA for supplying the moceuticals inc, Ridgefidd, Connecticut, USA for supplying the monoclonal antibodies. This work was supported by grants from the Medical Research Council, the West Midlands Regional
Health Authority, and the endowment fund of the former United Health Authority, and the
Birmingham Hospitals.

1 Mendenhall CL. Alcoholic hepatitis. In: Schiff L, Schiff ER, eds. Disease of the liver. Philadelphia: JB Lippicott Company, 1987: 669.

2 Sanchez-Tapias J, Thomas HC, Sherlock S. Lymphocyte populations in liver biopsy specimens from patients with populations in liver biopsy specimens from.
chronic liver disease. Gut 1977; 18: 472-5.

3 Dustin ML, Springer TA. Lymphocyte function-associated antigen-1 (LFA-1) interaction with intercellular adhesion molecule-1 (ICAM-1) is one of at least three mechanisms for lymphocyte adhesion to cultured endothelial cells. $\mathcal{F}$ Cell Biol 1988; 107: 321-31.

4 Dustin ML, Rothlein R, Bhan AK, Dinarello CA, Springer TA. Induction by IL 1 and interferon gamma:tissue distribution, biochemistry and function of a natural adherence molecule (ICAM-1). F Immunol 1986; 137: 245-54.

5 Buckle AM, Hogg $\mathrm{N}$. Human memory $T$ cells express intercellular adhesion molecule-1 which can be increased by interleukin 2 and interferon-g. Eur $\mathcal{F}$ Immunol 1990; 20: interleukin

6 Pober JS, Gimbrone MA, Lapierre LA, Mendrick DL, Fiers W, Rothlein R, et al. Overlapping patterns of activation of human endothelial cells by interleukin 1 , tumour necrosis factor and immune interferon. F Immunol 1986; 137: 1893-6. 7 Patarroyo M, Makgoba MW. Leucocyte adhesion to cells in immune and inflammatory responses. Lancet 1989; ii: $1139-$ 42.

8 Review by an International group. Alcoholic liver disease: morphological manifestations. Lancet 1981; i: 707-11.

9 Adams DH, Hubscher SG, Shaw J, Rothlein R, Neuberger $J M$. Intercellular adhesion molecule-1 on liver allograft during rejection. Lancet 1989; ii: $1122-4$.

10 Volpes R, Van Den Oord JJ, Desmet VJ. Immunohistochemical study of adhesion molecules in liver inflammation. Hepatology 1990; 12: 59-65.

11 Adams DH, Hubscher SG, Shaw J, Johnson GD, Babbs C, Rothlein R, et al. Increased expression of ICAM-1 on bile ducts in primary biliary cirrhosis and primary sclerosing cholangitis. Hepatology 1991; 14: 426-31.

2 Zetterman RK, Luisada-Opper A, Leevy CM. Alcoholic hepatitis. Cell-mediated immunological response to alcoholic hyalin. Gastroenterology 1976; 70: 382-4.

3 Kakumu S, Leevy CM. Lymphocyte cytoxicity in alcoholic hepatitis. Gastroenterology 1977; 72: 594-7.

14 Kanagasundaram N, Kakumu S, Chen T, Leevy C. Alcoholic hyalin antigen (AHAg) and antibody (AHAb) in alcoholic hepatitis. Gastroenterology 1977; 73: 1268-73.

15 McClain CJ, Cohen DA. Increased tumor necrosis factor production by monocytes in alcoholic hepatitis. Hepatology production by m

16 McClain CJ, Cohen DA, Dinarello CA, et al. Serum interleukin-1 (IL-1) activity in alcoholic hepatitis. Life Sci

17 Le J, Vilcek J. Tumor necrosis factor and interleukin-1: cytolines with multiple overlapping biological activities. $L a b$ Invest 1987; 56: 23448.

18 Nolan JP. Endotoxin, reticuloendothelial function and liver injury. Hepatology 1981; $1: 458-65$.

19 Chedid A, Mendenhall CL and the VACS 275. Enhanced expression of major histocompatibility complex (MHC) molecules and differential zonal distribution of lymphocytes in alcoholic liver disease. IASL Meeting, Gold Coast, Australia 1990. [Abstract]. Hepatology 1990; 12: 396.

20 Carithers RL, Franklin?, Herlong HF, Mae Diehl AM, Shaw EW, Combes B, et al. Methylprednisolone therapy in patients with severe alcoholic hepatitis. Ann Intern Med patients with seve 